\title{
THE LATERAL COMPLETION OF A COMPLETELY DISTRIBUTIVE LATTICE-ORDERED GROUP (REVISITED)
}

\author{
GARY DAVIS and STEPHEN H. McCLEARY
}

(Received 5 December 1978)

Communicated by E. Strzelecki

\begin{abstract}
The lateral completion of a completely distributive lattice-ordered permutation group is investigated via various completions, obtained by adjoining permutations which match some elements of the given group in various ways. This makes known results on the lateral completion of a completely distributive lattice-ordered group both transparent and easy.
\end{abstract}

1980 Mathematics subject classification (Amer. Math. Soc.): primary 06 F 15.

Ordinarily the lateral completion $G^{L}$ of a lattice-ordered group ( $l$-group) $G$ is explored by proceeding upward by induction on a tower of intermediate l-groups. For completely distributive l-groups, Byrd and Lloyd [8], following methods of Conrad [9], established the existence and uniqueness of the lateral completion. Bernau $[3,4]$ did the same for arbitrary $l$-groups, and found further properties of the lateral completion. Here we approach $G^{L}$ from above. We use a complete representation of $G$ as an $l$-permutation group $(G, S)$, (i.e. $S$ is a chain and $G$ is an $l$-subgroup of the $l$-group $A(S)$ of all order-preserving permuations of $S$ ), and let $G^{A}=\left\{h \in A(S): \forall s_{1}, \ldots, s_{n} \in S, \exists g \in G\right.$ such that $\left.s_{i} g=s_{i} h, i=1, \ldots, n\right\}$, an $l$-group recently investigated from a topological viewpoint by Ball [1], who also gave another proof of the existence and uniqueness of the lateral completion [2].

Inside $G^{A}$ lies a lateral completion of $G$, which (by virtue of Bernau's relatively easy proof that $G$ has at most one lateral completion) we may for convenience speak of as the lateral completion $G^{L}$. Except for uniqueness, these methods provide very easy proofs of all the standard properties of $G^{L}$, in most cases by establishing the corresponding properties of $G^{A}$. We find also that

OCopyright Australian Mathematical Society 1981 
intersection with $G$ gives an $o$-isomorphism from the partially ordered set of closed prime subgroups of $G^{L}$ onto that of $G$. This is done by means of another $l$-group $G^{C}$ intermediate between $G^{L}$ and $G^{A}$, which, unlike $G^{A}$, turns out to be independent of the representation of $G$. For transitive $(G, S)$, we obtain conditions under which $G^{L}$ is the wreath product of the lateral completions of the $o$-primitive components of $(G, S)$.

\section{\$1. Background}

An $l$-group $H$ is laterally complete if every (pairwise) disjoint set in $H$ has a supremum. An $l$-subgroup $G$ of $H$ is dense if for every $l<h \in H$, there exists $g \in G$ such that $1<g \leqslant h$; and density implies that if a subset of $G$ happens to have a supremum in $G$, say $g$, then $g$ is also its supremum in $H$. If $G$ is a dense $l$-subgroup of a laterally complete $l$-group $H$, and if there is no laterally complete $K$ such that $G \leqslant K<H$, then $H$ is called a lateral completion of $G$. ( $G<K$ means that $G$ is an $l$-subgroup of $K$.) If $G$ is a dense $l$-subgroup of a laterally complete $L$, it is easy to build inductively a lateral completion of $G$ inside $K$ (see [9]).

Although Bernau's proof that every $l$-group $G$ has a lateral completion is quite difficult, his proof [3, Theorem 3.6] that any two lateral completions of $G$ are $l$-isomorphic over $G$ is fairly easy-it consists essentially of his Lemma 3.4. This uniqueness was established earlier for completely distributive l-groups by Byrd and Lloyd [8], but for uniqueness alone, Bernau's proof is easier. Although our proofs do not depend on this uniqueness, we permit ourselves the convenience of taking it as known, and speak of the lateral completion $G^{L}$ rather than merely of $a$ lateral completion obtained from a given representation.

Most of the following information about $l$-permutation groups can be found in [10], as well as in the references cited.

The completely distributive $l$-groups $G$ are those for which the collection of prime subgroups $P$ which are closed (with respect to suprema of arbitrary-not necessarily disjoint - subsets of $P$ which happen to exist in $G$ ) have intersection \{1\} [7]. Any collection of closed primes having intersection $\{1\}$ yields a complete (preserving arbitrary suprema which exist in $G$ ) representation of $G$ as an $l$-subgroup of the $l$-group $A(S)$ of all automorphisms of some chain $S$ [6]. When $(G, S)$ is complete, all stabilizer subgroups $G_{s}, s \in S$ (equivalently, all stabilizers $G_{\bar{s}}, \bar{s} \in \bar{S}$ ) are closed primes of $G$ [15]. Here $G$ acts on $\bar{S}$, the completion by Dedekind cuts of $S$. Most of our results will deal directly with such representations, and the $l$-group $G^{A}$ will depend on the representation, though as mentioned above, $G^{L}$ will not. 
One says that sups are pointwise in $(G, S)$ if whenever $g=\sup _{i} g_{i}$, then $s g=\sup _{i} s g_{i}$ for each $s \in S$. (We shall reserve the notation " $V$ " for finite suprema.) It is almost true that whenever $(G, S)$ has closed stabilizers, sups are pointwise, and the following lemma and proposition remove the "almost" for those cases in which we shall be interested. As background which we shall not actually use, we mention that if $(G, S)$ is any given representation of a completely distributive $l$-group $G$, then there is a convex congruence $C$ such that $G$ acts faithfully on $S / C$, and $(G, S / C)$ has both closed stabilizers and pointwise sups [16].

LEMMA 1. Let $(G, S)$ be an l-permutation group having closed stabilizers, and suppose $\inf _{i} g_{i}=1$. Then this inf is pointwise at any $\bar{s} \in \bar{S}$ for which $\left\{\bar{s} g_{i}\right\}$ has a smallest element.

Proof. Let $\bar{s} g_{0}$ be the smallest element of $\left\{\overline{s g}_{i}\right\}$ and suppose by way of contradiction that $\bar{s}<\bar{s} g_{0}$. We have $g_{0}=g_{0} \cdot\left(\sup _{i} g_{i}^{-1}\right) \vee 1=\sup _{i}\left(g_{0} g_{i}^{-1} \vee 1\right)$. But each $g_{0} g_{i}^{-1} \vee 1 \in G_{\bar{s}}$, whereas $g_{0} \in G_{\tilde{s}}$, violating the fact that $G_{\bar{s}}$ is closed.

Proposition 2. Let $(G, S)$ be an l-permutation group having closed stabilizers. Then all disjoint sups in $G$ are pointwise.

This proposition follows from the dual of the lemma, and it implies that $G$ is an $\mathcal{E}$-subgroup of $A(\bar{S})$, that is, that for any disjoint supremum $g=\sup _{i G} g_{i}$, we also have $g=\sup _{i} A(\bar{S}) g_{i}$. As one is led to expect, whenever we construct a supremum for a disjoint set, the supremum will be constructed pointwise.

\section{Properties of the lateral completion $G^{L}$}

When $(G, S)$ is an $l$-permutation group, we shall be interested in the set $G^{A}$ of those elements $h$ of $A(S)$ satisfying the following condition:

(A) $h$ can be finitely matched at points of $S$ by elements of $G$, that is, for any $s_{1}, \ldots, s_{n} \in S$, there exists $g \in G$ such that $s_{i} g=s_{i} h, i=1, \ldots, n$.

Clearly $G<G^{A}<A(S)$, and by definition, $G^{A}$ respects the convex congruences of $(G, S)$. Ball discusses $G^{A}$ in [1], where it appears as the closure of $G$ relative to the "fine stabilizer topology" on $A(S)$. Here we maintain an algebraic setting. 
LEMMA 3. Let $(G, S)$ be any l-permutation group. Then $G^{A}$ is laterally complete.

Proof. Let $\left\{h_{i}: i \in I\right\}$ be a disjoint subset of $G^{A}$, and let $h$ be the pointwise supremum of $\left\{h_{i}: i \in I\right\}$. We show that $h \in G^{A}$. Let $s_{1}, \ldots, s_{n} \in S$. Let $I_{0}=\left\{i \in I\right.$ : $\operatorname{support}\left(h_{i}\right)$ contains some $\left.s_{j}\right\}$, a finite set. (Support $\left(h_{i}\right)$ means $\left\{s \in S \mid s h_{i} \neq s\right\}$.) Since the $h_{i}$ 's are disjoint, $\bigvee\left\{h_{i} \mid i \in I_{0}\right\}$ agrees with $h$ on $\left\{s_{1}, \ldots, s_{n}\right\}$. For each $i \in I_{0}$, pick $g_{i} \in G$ agreeing with $h_{i}$ on $\left\{s_{1}, \ldots, s_{n}\right\}$. Let $g=\bigvee\left\{g_{i}: i \in I_{0}\right\} \in G$. Then $g$ agrees on $\left\{s_{1}, \ldots, s_{n}\right\}$ with $\bigvee\left\{h_{i}: i \in I_{0}\right\}$ and thus also with $h$. Therefore $h \in G^{A}$, and certainly $h=\sup _{G^{A}}\left\{h_{i}: i \in I\right\}$.

Ball [1] used topological methods to prove the next lemma; here we give an algebraic proof.

LEMMA 4 (Ball). Let $(G, S)$ be any l-permutation group having closed stabilizers. Then $G$ is dense in $G^{A}$.

Proof. Let $1<h \in G^{A}$, and pick $s \in S$ such that $s<s h$. For each $t \in S$, pick $1<g_{t} \in G$ such that $s g_{t}=s h$ and $t g_{t}=t h$. Then for any lower bound $k$ of $\left\{g_{t}: t \in S\right\}$, we have $k<h$. Hence there exists $g \in G$ such that $1<g<h$, for otherwise $1=\inf _{G}\left\{g_{t}\right\}$, contradicting Lemma 1 .

THEOREM 5. Let $(G, S)$ be any l-permutation group having closed stabilizers. Then $G$ has a lateral completion inside $G^{A}$.

COROllary 6 (Byrd and Lloyd). Every completely distributive l-group has a lateral completion.

Bernau [4] has shown that for any l-group word $w\left(x_{1}, \ldots, x_{n}\right)$, the sentence " $\forall x_{1}, \ldots, x_{n}, w\left(x_{1}, \ldots, x_{n}\right)=1$ " holds in $G^{L}$ whenever it holds in $G$. Ball $[1,2]$ used topological methods to extend this result. In the present setting, even the extension is virtually trivial.

TheOREM 7 (Ball). Let $(G, S)$ have closed stabilizers. Let $\psi$ be any universally quantified disjunctions of conjunctions of atomic formulae " $w\left(c_{1}, \ldots, c_{m}, x_{1}, \ldots, x_{n}\right)=1 "$ where the l-group words $w$ may use constants $c_{1}, \ldots, c_{m}$ from $A(\bar{S})$. Then if $\psi$ holds in $G$, it holds also in $G^{A}$ and then in $G^{L}$.

Proof. Suppose $\psi$ fails in $G^{A}$. Pick elements $h_{i}$ of $G^{A}$ demonstrating this failure. For now, suppose that each $c_{i} \in A(S)$. Then for any given $h_{i}$, its contribution to the failure consists only of its effect on a finite number of points of $S$, and this effect can be matched by some $g_{i} \in G$. Hence $\psi$ fails in $G$. 
Coping with constants from $A(\bar{S})$ gets a bit tedious when dealing with all of $G^{A}$, and we omit the proof of this. However, there is no problem for $G^{L}$ : simply apply what has just been shown to $(G, \bar{S})$.

For any $l$-group $G,\left|G^{L}\right| \leqslant 2^{2^{|G|}} \cdot[3]$

Proposition 8. Let $G$ be completely distributive. Then $\left|G^{L}\right|<2^{|G|}$.

Proof. Since $G$ is completely distributive, the intersection of its closed primes is $\{1\}$. Thus for each $1 \neq g \in G$, we may pick a closed prime $P_{g}$ omitting $g$. Now we use this collection of closed primes to represent $G$ as an $l$-permutation group on a chain $S$ of cardinality $|G|$, with $(G, S)$ having closed stabilizers, $\left|G^{L}\right| \leqslant\left|G^{A}\right| \leqslant 2^{|S|}=2^{|G|}$. (Alternatively, this bound can be deduced from results in [8].)

The bound cannot be improved: the small sum $\Sigma_{i \in \mathbf{Z}} \mathbf{Z}_{i}$ of copies of the integers $\mathbf{Z}$ is a countable completely distributive $l$-group whose lateral completion $\Pi_{i \in \mathbf{Z}} \mathbf{Z}_{i}$ is uncountable.

Proposition 9. Let $(G, S)$ be an l-permutation group having closed stabilizers. Then $\left(G^{A}, S\right)$ also has closed stabilizers, and thus $G^{A}$ is completely distributive.

Proof. Let $H=G^{A}$. Suppose by way of contradiction that for some $\left\{h_{i}\right\} \subseteq$ $H_{s}, h=\sup _{i H} h_{i}$ but $s<s h$. Pick $g \in G$ such that $s g=s h$. Let $k_{i}=g h^{-1} h_{i} \in$ $H_{s}$. Then $g=g h^{-1} h=g h^{-1} \cdot \sup _{i H} h_{i}=\sup _{i H} g h^{-1} h_{i}=\sup _{i H} k_{i}$. For each $i$ and each $t \in S$, pick $g_{i, t}$ matching $k_{i}$ at $s$ and $t$. Then $g=\sup _{i, t}\left(g_{i, t} \wedge g\right)$, and we have $g_{i, c} \wedge g \in G_{s}$ but $g \notin G_{s}$, contradicting the fact that $(G, S)$ has closed stabilizers.

Since any dense $l$-subgroup of a completely distributive $l$-group is completely distributive, we have

Corollary 10 (Byrd and Lloyd). The lateral completion of a completely distributive l-group is completely distributive.

\section{Sharpening the description of the lateral completion}

EXAmple 11. Let $\mathbf{R}$ be the real line. An automorphism $h \in A(\mathbf{R})$ is piecewise linear if each $r \in \mathbf{R}$ has a neighbourhood $N_{r}$ such that the restriction to $N_{r}$ of the graph of $h$ consists of at most two line segments. Let $G$ consist of all piecewise linear $h \in A(\mathbf{R})$. Then $G^{L} \leqslant G^{A}=A(\mathbf{R})$, but as we shall see, $G^{L}$ is actually considerably smaller than $A(\mathbf{R})$. Let $H$ consist of those $h \in A(\mathbf{R})$ such that, for 
$\mathbf{R}_{h}$ the set of $r \in \mathbf{R}$ having no neighbourhood $N_{r}$ for which the restriction to $N_{r}$ of the graph of $h$ consists of at most two line segments, $\mathbf{R}_{h}$ is nowhere dense in $\mathbf{R}$; or equivalently, such that within each non-degenerate interval of $\mathbf{R}$, there exists a non-degenerate subinterval throughout which the graph of $g$ is a single line segment. Then $H$ is a laterally complete l-group containing $G$ as an $l$-subgroup. Hence $G^{L} \leqslant H$. We have been unable to decide whether $G^{L}=H$. ( $G^{L}$ does contain each $h \in A(\mathbf{R})$ which fixes all points in $\mathbf{R}_{h}$.) The l-groups $G^{D}$, $G^{E}$ and $G^{F}$ in the following discussion are motivated by the present example.

Given an $l$-permutation group $(G, S)$ having closed stabilizers, we explore several $l$-subgroups of $G^{A}$ within which the lateral completion $G^{L}$ must lie. For this purpose, we list several more conditions on automorphisms $h \in A(S)$.

(B) $h$ can be finitely matched at Dedekind cuts by elements of $G$, that is, for any $\bar{s}_{1}, \ldots, \bar{s}_{n} \in \bar{S}$, there exists $g \in G$ such that $\bar{s}_{i} g=\bar{s}_{i} h, i=1, \ldots, n$.

We shall say that $T \subseteq \bar{S}$ is a prime set of $(G, S)$ if $G_{T}=\left\{g \in G: \bar{t}_{g}=\bar{t}\right.$, $\forall \bar{t} \in T\}$ is a prime subgroup of $G$, or equivalently, if for any $\bar{t}, \bar{u} \in T, G_{\bar{t}}$ and $G_{\bar{u}}$ are comparable under inclusion. Any singleton $\{\bar{t}\}$ is a prime set, and if $T$ is a prime set and $g \in G$, then $T g$ is also a prime set. If $(G, S)$ has closed stabilizers, the closed primes of $G$ are precisely the $G_{T}$ 's for the various prime sets $T[13]$. Now for the next condition:

(C) $h$ can be finitely matched at prime sets of $(G, S)$, that is for any prime sets $T_{1}, \ldots, T_{n}$, there exists $g \in G$ such that $\bar{s} g=\bar{s} h$ for each $\bar{s} \in T_{1} \cup \cdots \cup T_{n}$.

A segment of a totally ordered set is a convex subset. A segment is non-degenerate if it contains more than one point, and is infinite if it contains infinitely many points. The next condition is concerned with segments not of $S$, but of orbits $\bar{s} G$ of $(G, \bar{S})$. It is especially useful when dealing with $l$-groups such as the one in Example 11.

(D) For $I_{p}$ a non-degenerate segment of the orbit $\bar{s}_{p} G$, $p=1, \ldots, n$, there exists $g \in G$ which agrees with $h$ on non-degenerate subsegments $J_{p} \subseteq I_{p}, p=1, \ldots, n$.

When $T$ is a prime set of $(G, S)$, the following rule defines a total order on the set $\{T g: g \in G\}$ of translates of $T: T g_{1}<T g_{2}$ if and only if $\overline{t g}_{1}<\overline{t g}_{2}$ for all $i \in T$. (This rule corrsponds to the usual total order on the set $R\left(G_{T}\right)$ of right cosets of the prime $G_{T}$.) 
(E) If $T_{p}$ is a prime set of $(G, S)$ and $I_{p}$ a non-degenerate segment of $\left\{T_{p} g: g \in G\right\}, p=1, \ldots, n$, then there exists $g \in G$ which agrees with $h$ on non-degenerate subsegments $J_{p} \subseteq I_{p}, p=1, \ldots, n$.

(F) For any set $I_{1}, \ldots, I_{n}$ of infinite segments of $S$, there exists $g \in G$ which agrees with $h$ on infinite subsegments $J_{1} \subseteq I_{1}, \ldots, J_{n} \subseteq I_{n}$.

Replacing "infinite" by "non-degenerate" in condition (F) would yield, in the presence of condition (A), a strictly weaker condition (whereas conditions (D) and (E) are unaffected by this distinction). For any of the three conditions, restricting consideration to pairwise disjoint $I_{1}, \ldots, I_{n}$ yields an equivalent condition.

For $X=A, B, C, D, E$, or $F$, we let $G^{X}=\{h \in A(S) \mid h$ satisfies condition $(X)\}$. Obviously $G^{C} \subseteq G^{B} \subseteq G^{A}$ and $G^{E} \subseteq G^{D}$. In fact no other containments hold among the $G^{X}$. This remains true even in the presence of condition (B), (for example, $G^{F} \cap G^{B} \underline{g} G^{C}$, and similarly whenever the right-hand side is not $G^{A}$ or $G^{B}$ ). Moreover, each of these non-containments can be demonstrated via a transitive $(G, S)$ except for " $G^{D} \ell G^{F}$ ". Most of the requisite examples are easy to construct; we mention here one which is not so easy.

EXAMPLE 12. Let $(K, U)$ be the wreath product $\operatorname{Wr}\left\{\left(\mathbf{Z}_{\gamma}, \mathbf{Z}_{\gamma}\right) ; \gamma \in \mathbf{Z}^{+}\right\}$of copies of the integers $Z$ indexed by $Z^{+}$. (See [12] for the definition of wreath product.) Let $H$ consist of those members $k$ of $K$ such that for a given $\gamma$, all components $\mathrm{ik}_{\gamma, u}$ are the same integer $k_{\gamma}$, and $k_{\gamma} \neq 0$ for only finitely many $\gamma$. Let $G$ be the subgroup of $(W, S)=(H, U) \mathrm{Wr}(\mathrm{Z}, \mathrm{Z})$ consisting of those members for which there is a single sequence $\left\{h_{i}: i \in \mathbf{Z}\right\}$ indexed by all of $\mathbf{Z}$ but having only finitely many non-zero entries, such that in the copy of $(H, U)$ corresponding to $j \in \mathbf{Z}$, the sequence $\left\{k_{\gamma}: \gamma \in \mathbf{Z}^{+}\right\}$is obtained by shifting $\left\{h_{i}\right.$ : $i \in \mathbf{Z}\} j$ places to the right and using only the part then indexed by $\mathbf{Z}^{+}$. This is an example from [15] in a different guise, with the group operation reversed.

$G$ is in fact a totally ordered group, so that $G=G^{L}$, and $G$ acts transitively on $S$ (because each $u \in U$ has only finitely many non-zero entries). Since $G$ is an $O$-group, $\{1\}$ is a closed prime, but no $G_{t}=\{1\}$. Since $G_{S}=\{1\}, S$ is a prime set, so $G^{c}=G$. However, any element of $W$ obtained by relaxing the requirement on the non-zero entries of $\left\{h_{i}: i \in \mathbf{Z}\right\}$ from "finite" to "well ordered" lies in $G^{B} \cap G^{D} \cap G^{F}$. 
Proposition 13. Let $(G, S)$ be an l-permutation group having closed stabilizers, and let $G^{Y}$ be the intersection of any collection of $G^{X}$ that includes $G^{A}$. Then $\left(G^{Y}, S\right)$ is also an l-permutation group having closed stabilizers. Moreover, $G$ is dense in $G^{Y}$, and $G^{Y}$ is laterally complete. Hence $G^{L}<G^{Y}$.

Proof. To verify that all $G^{X}$ are laterally complete $l$-groups, we claim that it suffices to consider $G^{D}$ and $G^{F}$. We have already treated $G^{A}$. The $G^{B}$ for $(G, S)$ coincides with the $G^{A}$ for $(G, \bar{S})$. The $G^{C}$ for $(G, S)$ is canonically $l$-isomorphic to the $G^{B}$ of $(G, U)$, the representation of $G$ obtained by attaching to one end of $S$ the chains $R\left(G_{T}\right)$ for the various non-singleton prime set $T$, and letting $G$ act on each $R\left(G_{T}\right)$ in the usual fashion. Finally, $G^{E}$ is related to $G^{D}$ as is $G^{C}$ to $G^{B}$.

Clearly $G^{B}$ and $G^{F}$ are $l$-subgroups of $A(S)$ and thus so is $G^{Y}, G$ is dense in $G^{A}$ and $G<G^{Y} \leqslant G^{A}$, so $G$ is dense in $G^{Y}$, and $G^{Y}$ is dense in $G^{A}$ and thus also has closed stabilizers.

Now we show that $G^{D}$ is laterally complete, modelling the argument after the proof of Lemma 3. Let $\left\{h_{i}: i \in I\right\}$ be a disjoint subset of $G^{D}$, and let $h$ be the pointwise supremum of $\left\{h_{i}: i \in I\right\}$. Let $I_{p}$ be a non-degenerate segment of the orbit $\bar{s}_{p} G, p=1, \ldots, n$. For each $p$, pick an $h_{i}$ whose support (in $\bar{S}$ ) meets $I_{p}$ and let $I_{p}^{\prime}=I_{p} \cap \operatorname{support}\left(h_{i}\right)$; or if there is no such $h_{i}$, let $I_{p}^{\prime}=I_{p}$. We arbitrarily order the finitely many $h_{i}$ thus picked. Since the first $h_{i} \in G^{D}$, we may pick $g_{i} \in G$ which agrees with $h_{i}$ on non-degenerate subsegments $I_{p}^{\prime \prime} \subseteq I_{p}^{\prime}$. This $g_{i}$ agrees with $h_{i}$ on those segments $I_{p}^{\prime \prime}$ for which $I_{p}^{\prime}$ meets (and thus is contained in) $\operatorname{support}\left(h_{i}\right)$, and $g_{i}$ acts like the identity on the other segments $I_{p}^{\prime \prime}$. For the second $h_{i}$ (which we denote by $h_{j}$ ), we may pick $g_{j} \in G$ which agrees with $h_{j}$ on non-degenerate subsegment;s $I_{p}^{\prime \prime \prime} \subseteq I_{p}^{\prime \prime \prime}$. Thus $g_{j}$ agrees with $h_{j}$ on those segments $I_{p}^{\prime \prime \prime}$ for which $I_{p}^{\prime}$ meets support $\left(h_{j}\right)$, and acts like the identity on the others. We proceed similarly through the remaining $h_{i}$. Let $g$ be the supremum of the finitely many $g_{i}$ thus obtained. Since the $h_{i}$ are disjoint, $g$ agrees with $h$ on the subintervals $I_{p}^{\prime \prime}{ }^{\prime \prime}$ obtained from the last $h_{i}$. Since $g \in G$, we have shown that $G^{D}$ is laterally complete. The same argument works for $G^{F}$.

Since the supremum produced above was the same (namely the pointwise supremum) for all $G^{X}, G^{Y}$ is also a laterally complete $l$-group.

THEOREM 14. Let $G$ be a completely distributive l-group. Then the l-groups $G^{C}$ and $G^{C} \cap G^{E}$ are independent of the representation, that is, any two versions of $G^{C}$ (or $G^{C} \cap G^{E}$ ) arising from complete representations of $G$ are l-isomorphic over $G$.

Proof. First we consider $G^{C}$. Let $(G, S)$ be any complete representation of $G$. Let $(G, U)$ be the representation obtained by splicing together in any order all 
chains $R(P)$, where $P$ runs over the collection of all closed primes of $G$, and letting $G$ act $\mathrm{n}$ the usual way. Let $G^{C}(S)$ and $G^{C}(U)$ denote the respective groups $G^{C}$.

When $P$ is a closed prime of $G$, let $T_{S}(P)$ be the prime set $\left\{\bar{s} \in S: P \subseteq P_{\bar{s}}\right\}$ so that $P=\cap\left\{G_{\bar{s}}: \bar{s} \in T_{S}(P)\right\}$.

For $h \in G^{C}(S)$, we define $\psi(h)$ to be the automorphism of $U$ induced by $h$, that is, for $P f \in U(P$ a closed prime of $G$, and $f \in G),(P f) \psi(h)=(P f) g$, where $g \in G$ is chosen to agree with $h$ on the prime set $T_{S}(P) f$. Such a $g$ exists because $h \in G^{C}(S)$, and if $g_{1}, g_{2} \in G$ agree on $T_{S}(P) f=T_{S}\left(f^{-1} P f\right)$, then $f^{-1} P f g_{1}=f^{-1} P f g_{2}$, and thus $P f g_{1}=P f g_{2}$. Clearly $\psi$ is an $l$-monomorphism from $G^{C}(S)$ into $G^{C}(U)$ which extends the "identity map" on $G$.

Now let $k \in G^{C}(U)$. Define $h: S \rightarrow S$ by setting $s h=s g$, where $g \in G$ is chosen so that in $R\left(G_{s}\right) \subseteq U, G_{s} g=G_{s} k$. Then more generally, for any $P f \in U$ such that $G_{P f}=G_{s}$, and for any $g \in G$ such that $(P f) g=(P f) k$, we have $s h=s g$; for $G_{s} g=G_{s} k$ by finite matching, since if $g_{1}, g_{2} \in G$ agree at $P f$, they also agree at $s$. It follows that $h$ is onto. For $r<s \in S$, there exists $g \in G$ such that $G_{r} g=G_{r} k$ and $G_{s} g=G_{s} k$, so that $r h=r g<s g=s h$. Hence $h \in A(S)$.

If $T$ is a prime set of $(G, S)$, then $T^{\prime}=\left\{G_{\bar{s}}: \bar{s} \in T\right\} \subseteq U$ is a prime set of $(G, U)$, and if $g \in G$ agrees with $k$ on $T^{\prime}$, then $g$ agrees with $h$ on $T$. (On $T \cap S$, this is an immediate consequence of the definition of $h$; and this definition of $h$ can be applied without change to $(G, \bar{S})$.) It follows that $h \in G^{c}(S)$.

Finally, we show that $\psi(h)=k$. Let $P f \in U$, where $P$ is a closed prime of $G$ and thus has the form $G_{T}\left(T=T_{s}(P)\right)$, and $f \in G$. Again, let $T^{\prime}=\left\{G_{\bar{s}}: \bar{s} \in T\right\}$. Then $T^{\prime} f$ is a prime set of $(G, U)$, and $G_{T^{\prime} f}=G_{P f}$. Pick $g \in G$ agreeing with $k$ on $T^{\prime} f$, so that since $G_{T^{\prime}}=G_{P f},(P f) g=(P f) k$. But as in the previous paragraph, $g$ agrees with $h$ on Tf. Hence $(P f) \psi(h)=(P f) g=(P f) k$, and thus $\psi(h)=k$.

This proves the theorem for $G^{C}$, and from that, the result for $G^{C} \cap G^{E}$ follows easily.

If $G<H$, if every non-trivial convex $l$-subgroup of $H$ meets $G$ non-trivially (which is automatic if $G$ is dense in $H$ ), and if $P \rightarrow P \cap G$ provides an 0 -isomorphism from the partially ordered set of closed primes of $H$ onto that of $G$, then $H$ is called an $N$-extension of $G$ [5]. If $H$ is an $N$-extension of $G$, and if $H$ is completely distributive, then the inverse of the restriction map $P \rightarrow P \cap G$ is given by $Q \rightarrow \bar{Q}$, where $Q$ is a closed prime of $G$ and $\bar{Q}$ denotes its closure in $H$. (It suffices to show that each $\bar{Q}$ is prime in $H$. Let $P$ be the closed prime of $H$ such that $P \cap G=Q$, and suppose by way of contradiction that $P$ contains $\bar{Q}$ properly. As a closed convex $l$-subgroup of a completely distributive $H, \bar{Q}$ is the intersection of some collection of closed primes of $H$ [13], so we may pick a 
closed prime $P^{\prime}$ of $H$ such that $\bar{Q} \subseteq P$ but $P \nsubseteq P^{\prime}$. Then $P \cap G=Q \subseteq P^{\prime} \cap$ $G$, a contadiction.)

Byrd and Lloyd [7] showed that when $G$ is completely distributive, the map $P \rightarrow P \cap G$ is one-to-one from the set of closed primes of $G^{L}$ into that of $G$. Here we find that $G^{L}$ is actually an $N$-extension of $G$.

Lemma 15. Let $K$ be an $N$-extension of $G$, with $K$ completely distributive; and let $G<H<K$, with $H$ dense in $K$. Then $K$ is an $N$-extension of $H$ and $H$ is an $N$-extension of $G$.

Proof. Since $H$ is dense in $K$, the intersection with $H$ of any closed prime of $K$ is closed in $H$. We shall show that every closed prime of $H$ is in the intersection with $H$ of some closed prime of $K$; the rest of the proof is straightforward. Since $K$ is completely distributive, we can obtain a representation $(K, S)$ with closed stabilizers. Let $P$ be a closed prime of $H$, say $P=H_{T}$, where $T \subseteq \bar{S}$. Then $K_{T}$ is a closed prime of $K$ for which $K_{T} \cap H=H_{T}$, provided that whenever $H_{\bar{t}} \subseteq H_{\bar{u}}(\bar{t}, \bar{u} \in T)$, we have $K_{\bar{i}} \subseteq H_{\bar{u}}$. But if $H_{\bar{i}} \subseteq H_{\bar{u}}$, then $G_{\bar{t}} \subseteq G_{\bar{u}}$. The fact that $K$ is an $N$-extension of $G$ tells us first that $G_{\bar{t}}=K_{\bar{t}} \cap G$ and $G_{\bar{u}}=K_{\bar{u}} \cap G$ are closed in $G$, and then that $K_{\bar{i}} \subseteq K_{\bar{u}}$, as desired.

Lemma 16. Let $(G, S)$ have closed stabilizers. Then $G^{C}$ is an $N$-extension of $G$.

In fact, $G^{A}$ and $G^{B}$ are also $N$-extensions of $G$, but we omit the proof of that.

Proof. $\left(G^{C}, S\right)$ has closed stabilizers by Proposition 13. If $G_{T}^{C}$ is an (arbitrary) closed prime of $G^{C}$, then $G_{T}^{C} \cap G=G_{T}$ is a closed prime of $G$, and if $G^{T}<G_{U}$, then by condition (C), $G_{T}^{C}<G_{U}^{C}$. Moreover, if $G_{T}$ is an (arbitrary) closed prime of $G$, then for $\bar{t}, \bar{u} \in T$, we have either $G_{\bar{t}}<G_{\bar{u}}$ and thus $G_{\bar{i}}^{C}<G_{\bar{u}}^{C}$, or else $G_{\bar{u}} \leqslant G_{\bar{t}}$ and thus $G_{\bar{u}}^{C} \leqslant G_{\bar{t}}^{C}$; so that $G_{T}^{C}$ is a (closed) prime of $G^{C}$ for which $G_{T}^{C} \cap G=G_{T}$.

ThEOREM 17. Let $G$ be completely distributive. Then its lateral completion $G^{L}$ is an $\mathrm{N}$-extension of $\mathrm{G}$.

However, $G^{L}$ need not be an $a^{*}$-extension of $G$ (that is, preserve closed convex $l$-subgroups). Let $(H, S)$ be the wreath product $(A(\mathbf{R}), \mathbf{R}) \operatorname{Wr}(A(\mathbf{R}), \mathbf{R})$, and $G$ the $l$-subgroup $\left\{\left(\left\{g_{r}\right\}, g\right) \in H\right.$ : $\operatorname{support}(g)$ is bounded, and for sufficiently large $r, g_{r}=g$ \}. Then $G^{L}=H$; the reader may verify this himself or await Theorem 19. But $H$ is not an $a^{*}$-extension of $G$ [11, Example 4.11]. 
Byrd and Lloyd [8] also established some other results about completely distributive $l$-groups $G$ which we can easily recover here. For example, if $P$ is any closed prime of $G^{L}$, then $P G=G^{L}$ (so that the natural embedding $P g \rightarrow \overline{P g}$ of $G / P$ into $G^{L} / \bar{P}$ is onto). In our present framework, this just says that each $h \in G^{L}$ can be matched at $P$ by some $g \in G$, which is of course true for $G^{C}$ and thus also for $G^{L}$. If $\left\{Q_{i}\right\}$ is a closed kernel of $G$ (that is, if the intersection of all conjugates in $G$ of all $Q_{i}$ is $\left.\{1\}\right)$, then $\left\{\overline{Q_{i}}\right\}$ is a closed kernel of $G^{L}$. This can be seen by representing $G$ via $\left\{Q_{i}\right\}$ and recalling that $G^{L}<G^{A}$. If $\left\{Q_{i}\right\}$ is an invariant set of closed primes of $G$, then $\left\{\bar{Q}_{i}\right\}$ is invariant in $G^{L}$. To see this, enlarge $\left\{Q_{i}\right\}$ to a closed kernel of $G$ and proceed as above, using the fact that $h^{-1} H_{s} h=H_{s h}$.

\section{Relation to the structure of transitive groups}

In this section we assume familiarity with the structure of transitive l-permutation groups [12]. Let us see first what our results say about lateral completions of transitive 0-primitive l-permutation groups $(G, S)$. Recall the three types of such groups [14]:

(1) $(G, S)$ is the regular representation of a subgroup of the real numbers $\mathbf{R}$.

(2) $(G, S)$ is 0-2-transitive, that is for all $r<s$ and $t<u$ in $S$, there exists $g \in G$ such that $r g=t$ and $s g=s .(G, S)$ is pathological if it contains no non-identity element whose support is bounded.

(3) $(G, S)$ is periodic with period $z \in A(\bar{S})$, with the centralizer in $A(\bar{S})$ of $G$ is generated as a group by some $1<z \in A(\bar{S})$.

The observations in this paragraph and the next which stem from the containment $G^{L} \leqslant G^{A}$ are due to Ball [1]. If $(G, S)$ is regular, then of course $G=G^{L}=G^{A}$. If $(G, S)$ is pathologically 0-2-transitive, the one case in which stabilizers are not closed [15], the present results do not apply. If $(G, S)$ is non-pathologically $0-2$-transitive, then since in fact it is even 0 - $n$-transitive, we unfortunately have $G^{A}=A(S)$; but $G^{L}<G^{B} \cap G^{D}$, which is often helpful in further pinning down $G^{L}$. (When $G$ is 0 -primitive, every closed prime is a stabilizer $G_{\bar{s}}$, so $G^{C}$ and $G^{E}$ make no further contribution.) If $(G, S)$ has period $z \in A(\bar{S})$, then $G^{L}<G^{A}<\{h \in A(S) \mid h z=z h\}$ by Theorem 7 applied to the constant $z \in A(\bar{S})$, so that $G^{L}$ has the same period as $G$. The reader familiar with periodic groups will observe easily that in fact $G^{A}=\{h \in A(S) \mid h z=z h\}$. Again, $G^{L}<G^{B} \cap G^{D}$.

Now let $(G, S)$ be any transitive $l$-permutation group having closed stabilizers, so that $G \leqslant G^{L} \leqslant A(S)$. $\left(G^{L}, S\right)$ has the same tower $\Gamma$ of covering pairs of convex congruences as does $(G, S)$. Moreover, for each $\gamma \in \Gamma$, the 0-primitive 
component $\left(G_{\gamma}^{L}, S_{\gamma}\right)$ is related to $\left(G_{\gamma}, S_{\gamma}\right)$ as in the previous paragraph, that is, $G_{\gamma}^{L}<G_{\gamma}^{B} \cap G_{\gamma}^{D}$. (If $\left(G_{\gamma}, S_{\gamma}\right)$ is pathological, we can only say that $G_{\gamma}^{L}<A\left(S_{\gamma}\right)$; and indeed equality can obtain.) The reader familiar with intransitive structure theory [16] will observe that these remarks apply equally well to intransitive groups.

Proposition 18. Let $W$ be a wreath product of transitive 0-primitive l-permutation groups which are laterally complete and are non-pathological. Then $W$ is laterally complete.

Proof. The factors $\left(G_{\gamma}, S_{\gamma}\right)$ have closed stabilizers, so all disjoint sups in $G_{\gamma}$ are pointwise.

It may be that pathologically 0-2-transitive groups are never laterally complete (see Section 5), but we have been unable to show this.

TheOREM 19. Let $(G, S)$ be a transitive l-permutation group whose tower of convex congruences is well ordered, and whose 0-primitive components $\left(G_{\gamma}, S_{\gamma}\right)$ are non-pathological. Suppose also that for any covering pair $\mathcal{E}<\mathcal{F}$ of convex congruences of $(G, S)$, there exists $g \in G$ which fixes all $\mathcal{E}$-classes outside some $\mathscr{F}$-class $B$ while moving some $\mathcal{E}$-class within $B$. Then

$$
G^{L}=\operatorname{Wr}\left\{\left(\left(G_{\gamma}\right)^{L}, S_{\gamma}\right) \mid \gamma \in \Gamma(G, S)\right\} .
$$

The hypotheses of the theorem are satisfied by wreath products (restricted or unrestricted) for which the index set is well ordered and the 0-primitive components are arbitrary non-pathological 0-primitive groups. More interestingly, they are satisfied by the group $(G, S)$ following Theorem 17 . To prove Theorem 19, we need a couple of lemmas.

LEMMA 20. Let $(G, S)$ be non-pathologically 0-2-transitive, and let $B$ be the set of elements having bounded support. Then $B^{L}=G^{L}$.

Proor. Since $B$ is dense in $G, B^{L}<G^{L}$. It suffices now to show $G \subseteq B^{L}$. First, let $1<g \in G$ have only one (unbounded) interval of support, and pick any $r<s<t<u$ from support $(g)$. We claim that there exists $b \in B$ agreeing with $g$ on the interval $(s, t)$ with support contained in $(r, u)$. Any $1<b^{\prime} \in B$ has a conjugate $b^{\prime \prime}$ such that $s b^{\prime \prime}=\operatorname{tg}$ and $\operatorname{support}\left(b^{\prime \prime}\right) \subseteq(r, u)$, and we simply take $b=g \wedge g^{\prime \prime}$. Now, for each integer $n$, pick $b_{n} \in B$ agreeing with $g$ on $\left(s g^{n}, s g^{n+1}\right)$ and supported by $\left(s g^{n-1}, s g^{n+2}\right)$. For $i=0,1,2$, the set $\left\{b_{n}: n \equiv i\right.$ $(\bmod 3)\}$ is disjoint, so $B^{L}$ contains its pointwise supremum $g_{i}$. Hence $B^{L}$ contains $g_{1} \vee g_{2} \vee g_{3}=g$. It follows easily that $B^{L} \supseteq G$. 
Lemma 21. Let $\delta_{\gamma}<\delta^{\gamma}$ be a covering pair of convex congruences of $(G, S)$, and let $B$ be any $\delta^{\gamma}$-class. Let $H$ consist of the actions on $B / \delta_{\gamma}$ of $\{g \in G \mid g$ fixes all $\delta_{\gamma}$-classes outside $\left.B\right\}$, and suppose $H \neq\{1\}$. Then $H=G_{\gamma}$ unless $\left(G_{\gamma}, S_{\gamma}\right)$ is 0-2-transitive, and in that case $H$ contains all elements of $G_{\gamma}$ having bounded support.

The proof of the lemma is similar to that of [15, Lemma 16], and we omit it.

Proof of Theorem 19. We think of $(G, S)$ as being embedded in its wreath product $\operatorname{Wr}\left\{\left(G_{\gamma}, S_{\gamma}\right) \mid \gamma \in \Gamma=\Gamma(G, S)\right\}$. Since $\Gamma$ is well ordered, the chain on which this wreath product acts is simply $S$ itself. Since the 0-primitive components of $(G, S)$ are non-pathological, they have closed stabilizers, and thus $\left(G_{\gamma}\right)^{L} \leqslant A\left(S_{\gamma}\right)$. Hence the wreath product $W=\operatorname{Wr}\left\{\left(\left(G_{\gamma}\right)^{L}, S_{\gamma}\right) \mid \gamma \in \Gamma\right\}$ also acts on $S$, and $G \leqslant W$.

To show that $G$ is dense in $W$, it suffices to show that each $1<w \in W$ supported by a single primitive segment $B$ of $S$ exceeds some $1<g \in G$. Let $\left(G_{\delta}, S_{\delta}\right)$ be the bottom 0-primitive component of $(G, S)$. The restriction $W \mid B$ lies in $\left(G_{\delta}\right)^{L}$ and thus exceeds some $1<h \mid B$ (with $h \in G$ and $B h=B$ ). By Lemma 21, there exists $k \in G$ supported by $B$ such that $1<k|B<h| B<w \mid B$. Hence $1<k \leqslant w$, establishing the density of $G$ in $W$. By Proposition $18, W$ is laterally complete. Hence $G \leqslant G^{L}<W$.

$(W, S)$, like any wreath product of transitive 0-primitive groups which is not locally pathological, has closed stabilizers [15, Corollary 18], so all disjoint suprema in $W$ are pointwise. Since $G$ is dense in $W$, all disjoint suprema in $G^{L}$ are also suprema in $W$ and thus are pointwise.

Now we suppose that for a given convex congruence $\mathscr{E}, G^{L}$ contains each $h \in W$ which fixes all $\mathcal{E}$-classes, and prove that this is also true for the convex congruence $\mathscr{F}$ which covers $\mathcal{E}$.

Let $B$ be any $\mathscr{F}$-class, and let $K=K_{B}$ consist of the set of actions on $B / \mathcal{E}$ of elements of $G^{L}$ which fix all $\varepsilon$-classes outside $B$. By hypothesis, $K \neq\{1\}$, so $K$ fits the description of Lemma 21 . Using the induction hypothesis, we see that $K$ is closed under (pointwise) disjoint suprema. By Lemma 20, $K$ fills out all of the $\gamma$ th 0-primitive component $\left(G_{\gamma}\right)^{L}$ of $W$. In particular, for any $f \in W$ supported by $B$, the action of $f$ on $B / \mathcal{E}$ is matched by the action on $B / \mathcal{E}$ of some $d \in G^{L}$ which fixes all $\mathscr{E}$-classes outside $B$. Then $f d^{-1}$ fixes all $\mathscr{E}$-classes, so $f d^{-1} \in G^{L}$ by induction, and thus $f \in G^{L}$.

But now $G^{L}$ must contain each $h \in W$ which fixes all $\mathscr{F}$-classes. (Write $h$ as the disjoint supremum of elements supported by individual $\mathcal{F}$-classes.) By induction, $G^{L}$ contains every $h \in W$ which, for some convex congruence $\mathcal{E}$ of $(G, S)$ fixes all $E$-classes. 
Hence $G^{L}$ contains each $h \in W$ having precisely one supporting interval. (Even if that interval is unbounded, if we pick any $s$ in the interval and let $\mathcal{E}$ be large enough that $s$ and $s h$ are congruent $\bmod \delta$, then $h$ fixes all $\mathcal{E}$-classes.) It follows that $G^{L}$ contains $W$, concluding the proof.

\section{Non-extendability of the present methods}

Complete distributivity is essential for our methods. Let $G=\{h \in A(\mathbf{R}) \mid \exists m$ $=m_{h} \in \mathbf{Z}^{+}$such that $\left.\forall r \in \mathbf{R},(r+m) h=r h+m\right\}$, the usual pathologically 0 -2-transitive $l$-permutation group. We shall show that $G^{L}$ does not lie in $A(\mathbf{R})$.

To do this, we give a disjoint set $\left\{g_{n}: n \in \mathbf{Z}^{+}\right\}$of $G$ such that there is no $H$ for which $G \leqslant H \leqslant A(\mathbf{R}), \sup \left\{g_{n}\right\}$ exists in $H$, and $G$ is dense in $H$. Let the graph of $f_{n} \in A(\mathbf{R})$ include the line segment whose end points are $\left(-\frac{1}{2},-\frac{1}{2}\right)$ and $\left(0, \frac{1}{4}\right)$, and the line segment whose end points are $\left(0, \frac{1}{4}\right)$ and $\left(\frac{1}{4}+\frac{1}{4} n, \frac{1}{4}+\frac{1}{4} n\right)$; and let $f_{n}$ act like the identity elsewhere. Let $g_{n} \in G$ be the pointwise supremum of the set $\left\{z^{-\left(2^{n-1}+2^{n} k\right)} f_{n} z^{2^{n-1}+2^{n} k}: k \in \mathbf{Z}\right\}$, where $z$ is the transition $r \rightarrow r+1$. Thus the graph of $g_{n}$ has "bumps" like that of $f_{n}$, but with the "corners" at the integers $2^{n-1}+2^{n} k(k \in \mathbf{Z})$. The set $\left\{g_{n}: n \in \mathbf{Z}^{+}\right\}$is disjoint.

Suppose that in some $H, G \leqslant H \leqslant A(\mathbf{R}),\left\{g_{n}\right\}$ has a supremum $h$. First we consider the case in which $0 h \geqslant \frac{1}{4}$. Then $\frac{1}{8} h>\frac{1}{4}$, so for all $n$ greater than some sufficiently large $N, \frac{1}{8} f_{n}<\frac{1}{8} h$. Let $h^{\prime} \in G$ be the pointwise supremum of the set $\left\{z^{-2^{N-1} k} f_{N} z^{2^{N-1} k}: k \in \mathbf{Z}\right\}$, and let $h^{\prime \prime}=g_{1} \vee g_{2} \vee \cdots \vee g_{N-1} \vee h^{\prime} \in G$. Then $g_{n}<h^{\prime \prime}$ for all $n \in Z^{+}$, but $h \notin h^{\prime \prime}$ because $\frac{1}{8} h>\frac{1}{8} f_{N}=\frac{1}{8} h^{\prime \prime}$. This contradiction disposes of the first case and leaves us with the second, namely $0 h<\frac{1}{4}$. This time we pick $h^{\prime} \in G$ such that $0 h<0 h^{\prime}<\frac{1}{4}$, and such that $z h=h z$. Then there is some negative number $r$ sufficiently close to 0 that $h^{\prime} h^{-1} \vee 1$ moves strictly up all points in the intervalv $(r, 0)$, but leaves fixed all points in each interval $(n-r, n), 0 \neq h \in \mathbf{Z}$. Now pick $1<g^{\prime} \in G$ such that $z g^{\prime}=g^{\prime} z, g$ moves some point in $(r, 0)$, and the support of $g^{\prime}$ is contained in the union of the intervals $(n-v, n) n \in \mathbf{Z}$. Then $1<h^{\prime} h^{-1} \vee 1 \wedge g^{\prime} \in H$ has support contained in $(r, 0)$ and thus does not exceed any $1<g \in G$, so that $G$ is not dense in $H$.

\section{References}

[1] R. Ball, 'Topological lattice ordered groups', to appear.

[2] , 'Convergence and Cauchy structures on lattice ordered groups', to appear.

[3] S. J. Bernau, 'The lateral completion of an arbitrary lattice group', J. Austral. Math. Soc. Ser. A 19 (1975), 263-289. 
[4] _ 'Varieties of lattice groups are closed under E-completion', Symposia Math. 21 (1977), 349-355.

[5] R. Bleier and P. Conrad, The lattice of closed ideals and $a^{*}$-extensions of an abelian $l$-group, Pacific J. Math. 47 (1973), 329-340.

[6] R. D. Byrd, 'Complete distributivity in lattice-ordered groups', Pacific J. Math. 20 (1967), 423-432.

[7] R. D. Byrd and J. T. Lloyd, 'Closed subgroups and complete distributivity in lattice-ordered groups', Math. Zeit 101 (1967), 123-130.

[8] _ A note on lateral completions in lattice-ordered groups, J. London Math. Soc. (2) (1969), 358-362.

[9] P. Conrad, 'The lateral completion of a lattice-ordered group', Proc. London Math. Sac. (3) 19 (1969), 444-480.

[10] A. M. W. Glass, Ordered permutation groups (Bowling Green State University, Bowling Green, Ohio, 1976).

[11] A. M. W. Glass, W. C. Holland and S. H. McClearly, ' $a$ *-closures of completely distributive lattice-ordered groups', Pacific J. Math. 59 (1975), 43-67.

[12] W. C. Holland and S. H. McCleary, 'Wreath products of ordered permutation groups', Pacific J. Math. 31 (1969), 703-716.

[13] S. H. McClearly, 'The closed prime subgroups of certain ordered permutation groups', Pacific J. Math. 31 (1969), 745-753.

[14] _ ' '-primitive ordered permutation groups', Pacific J. Math. 40 (1972), 349-372.

[15] , 'Closed subgroups of lattice-ordered permutation groups', Trans. Amer. Math. Soc. 173 (1972), 303-314.

[16] _ The structure of intransitive ordered permutation groups, Algebra Universalis 6 (1976), 229-255.

La Trobe University

Bundoora

Victoria

Australia
University of Georgia

Athens

Georgia

U.S.A. 